\title{
Global Minimum for Curvature Penalized Minimal Path Method
}

\section{Da CHEN}

chenda@ceremade.dauphine.fr

Jean-Marie MIREBEAU

mirebeau@ceremade.dauphine.fr

Laurent D. COHEN

cohen@ceremade.dauphine.fr
Université Paris Dauphine,

CNRS, CEREMADE, UMR7534

F-75016, Paris, France
Minimal path problem [1] is posed on a bounded domain $\Omega$ and a metric $\mathcal{F}$ prescribing norm $\mathcal{F}_{x}$ (potentially asymmetric) at each point $x \in \Omega$. Let $\mathfrak{I}$ denote the collection of Lipschitz paths $\gamma:[0,1] \rightarrow \Omega$, and the path length $\ell_{\mathcal{F}}$ can be measured through the metric $\mathcal{F}$ as:

$$
\ell_{\mathcal{F}}(\gamma)=\int_{0}^{1} \mathcal{F}_{\gamma(t)}(\dot{\gamma}(t)) d t
$$

where $\dot{\gamma}(t)=\frac{d}{d t} \gamma(t)$. The minimal action map $\mathcal{U}$ from a set of initial source points $\mathcal{W}$, is the minimal energy of any path joining $x \in \Omega$ to $\mathcal{W}$ :

$$
\mathcal{U}(x):=\min \left\{\ell_{\mathcal{F}}(\gamma) ; \gamma \in \mathfrak{I}, \gamma(1)=x, \gamma(0) \in \mathcal{W}\right\}
$$

The minimal action map $\mathcal{U}$ is the unique viscosity solution to an Eikonal PDE, defined in terms of the dual metric $\mathcal{F}^{*}$. For all $x \in \Omega$ one has $\mathcal{U}(x)=$ 0 if $x \in \mathcal{W}$ and otherwise

$$
\mathcal{F}_{x}^{*}(-\nabla \mathcal{U}(x))=1, \quad \text { where } \mathcal{F}_{x}^{*}(u)=\sup _{v \neq 0} \frac{\langle u, v\rangle}{\mathcal{F}_{x}(v)} .
$$

The metric $\mathcal{F}$ considered in this paper combine a symmetric part, defined in terms of a symmetric positive tensor field $\mathcal{M}$, and an asymmetric part involving a vector field $\omega$ :

$$
\mathcal{F}_{x}(u)=\sqrt{\langle u, \mathcal{M}(x) u\rangle}-\langle\omega(x), u\rangle .
$$

We require $\left\langle\omega(x), \mathcal{M}^{1} u\right\rangle<1$ to ensure the metric positivity. In this work, we show that the Euler's elastic bending energy can be approximated by the Finsler metric with the form of (4).

Following Mumford [4], the weighted Euler's elasticas, minimizing the following bending energy:

$$
\mathcal{L}(\Gamma)=\int_{0}^{L}\left(\frac{1}{\alpha(s)}+\frac{1}{\beta(s)} \kappa^{2}(s)\right) d s
$$

$L$ denotes the classical curve length, $s$ is the arc-length parameter, and $\Gamma:[0, L] \rightarrow \Omega$ is a curve with non-vanishing velocity vector. $\kappa$ is the curvature and $\alpha, \beta$ are two positively weighted functions computed by the optimally oriented flux filter [2].

Our first step is to cast the elastica energy (5) in the form of path length with respect to a degenerate Finsler metric. For that purpose, let $S^{1}=[0,2 \pi[$ be the space of angles, with periodic boundary conditions, and for each angle $\theta$ let $\vec{v}_{\theta}=(\cos \theta, \sin \theta)$ be the corresponding unit vector. For $\gamma=(\Gamma, \theta) \in \Omega \times S^{1}$ and $\dot{\gamma}=(\dot{\Gamma}, \dot{\theta}) \in \mathbb{R}^{2} \times \mathbb{R}^{1}$ we can define

$$
\mathcal{F}_{\gamma}^{\infty}:= \begin{cases}\|\dot{\Gamma}\|+|\dot{\theta}|^{2} /\|\dot{\Gamma}\| & \text { if } \dot{\Gamma} \text { is positively proportional with } \vec{v}_{\theta} \\ \infty & \text { otherwise. }\end{cases}
$$

Consider an curve $\Gamma$ parametrized by arc-length, and its orientation lifting $\gamma=(\Gamma, \theta)$, with $\dot{\Gamma}(s):=\vec{v}_{\theta}$. By assumption one has $\|\dot{\Gamma}(s)\|=1$, hence $\ddot{\Gamma}=\kappa(s) \dot{\Gamma}(s)^{\perp}$, so that $\kappa(s)=\dot{\theta}(s)$ and

$$
\int_{0}^{L}\left(1+\kappa^{2}(s)\right) d s=\int_{0}^{L} \mathcal{F}_{\gamma(s)}^{\infty}(\dot{\gamma}(s)) d s .
$$

The weights $\alpha, \beta$ of (5) are easily taken into account, by modifying (6) approximately. Note that thanks to the homogeneity of $\mathcal{F}_{\gamma}^{\infty}(\dot{)}$, the right hand side of (7) is invariant under reparametrizations of the lifted path $\gamma$. However, the degenerate metric $\mathcal{F}^{\infty}$ is too singular to apply the fast marching algorithm directly, hence we introduce a family tamings depending on a penalization parameter $\lambda \gg 1$ :

$$
\mathcal{F}_{\gamma}^{\lambda}(\dot{\gamma}):=\sqrt{\lambda^{2}\|\dot{\Gamma}\|^{2}+2 \lambda|\dot{\theta}|^{2}}-(\lambda-1)\left\langle\vec{v}_{\theta}, \dot{\Gamma}\right\rangle
$$

Again, the weights $\alpha, \beta$ can easily be incorporated into the metric $\mathcal{F}^{\lambda}$. As $\lambda \rightarrow \infty$ one has:

$$
\begin{aligned}
\mathcal{F}_{\gamma}^{\lambda}(\dot{\gamma}) & =\lambda\|\dot{\Gamma}\| \sqrt{1+\frac{2|\dot{\theta}|^{2}}{\lambda\|\dot{\Gamma}\|^{2}}}-(\lambda-1)\left\langle\vec{v}_{\theta}, \dot{\Gamma}\right\rangle \\
& =\lambda\|\dot{\Gamma}\|\left(1+\frac{|\dot{\theta}|^{2}}{\lambda\|\dot{\Gamma}\|^{2}}+\mathcal{O}\left(\frac{1}{\lambda^{2}}\right)\right)-(\lambda-1)\left\langle\vec{v}_{\theta} \dot{\Gamma}\right\rangle \\
& =\|\dot{\Gamma}\|+\frac{|\dot{\theta}|^{2}}{\|\dot{\Gamma}\|}+(\lambda-1)\left(\|\dot{\Gamma}\|-\left\langle\vec{v}_{\theta}, \dot{\Gamma}\right\rangle\right)+\mathcal{O}\left(\frac{1}{\lambda}\right)
\end{aligned}
$$

which tends to $\mathcal{F}_{\lambda}^{\infty}(\dot{\gamma})$ as $\lambda \rightarrow \infty$. The metric $\mathcal{F}^{\lambda}$ has precisely the required form (4), with a diagonal tensor field $\mathcal{M}$ :

$$
\mathcal{M}=\left(\begin{array}{ccc}
\lambda^{2} & 0 & 0 \\
0 & \lambda^{2} & 0 \\
0 & 0 & 2 \lambda
\end{array}\right)
$$

and a non-zero vector field $\omega(\gamma, \theta):=\vec{v}_{\theta}$. Then the minimal action map $\mathcal{U}^{\lambda}$ associated to the metric $\mathcal{F}^{\lambda}$ can be efficiently computed with the fast marching method [3].

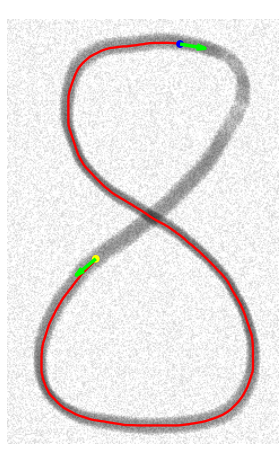

(a)

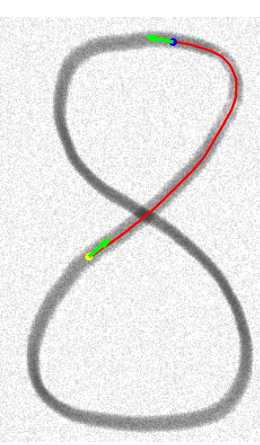

(b)

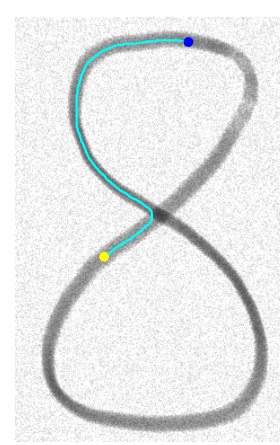

(c)
Figure 1: Comparison results on synthetic images. Yellow points denote the initial source point and blue points denote the endpoints. Green arrows in (a) and (b) demonstrate the initial and end tangent vectors for the proposed algorithm.

In Fig. 1, we show the extraction results (Fig. 1(a) and (b) ) from the proposed minimal path model and the Cohen-Kimmel model [1](Fig. 1(c)). In (Fig. 1(a) and (b) ), given different initial and end tangent vector as indicated by the green arrows, one could get different extraction results (shown as red solid curves) due to the asymmetric Finsler metric. Especially in Fig. 1(a), the curve length of the extracted geodesic is much longer than that in Fig. 1(b). With the proposed Finsler metric based curvature-penalty minimal path model, the proposed model can choose a correct path. For the Cohen-Kimmel model, the extracted path only follows the way which owns the largest speed values instead of choosing a smooth path.

[1] L. D. Cohen and R. Kimmel. Global minimum for active contour models: A minimal path approach. International Journal of Computer Vision, 24(1):57-78, 1997.

[2] Max W. K. Law and Albert C. S. Chung. Three Dimensional Curvilinear Structure Detection Using Optimally Oriented Flux. Computer Vision-ECCV 2008. Springer Berlin Heidelberg, pages 368382, 2008.

[3] Jean-Marie Mirebeau. Efficient fast marching with Finsler metrics. Numerische Mathematik, 126(3):515-557, 2014.

[4] David Mumford. Elastica and computer vision. Springer, 1994. 